\title{
SPACE-AIR-GROUND CO-OBSERVATION IN WATERSHED MANAGEMENT: THE ESTABLISHMENT OF SYSTEM
}

\author{
Liang Zhong ${ }^{1 *}$,Jingmin $\mathrm{Yu}^{1}$,Xuan Tang ${ }^{2}$, S. Pan ${ }^{3}$ \\ ${ }^{1}$ Changjiang Spatial Information Technology Engineering Co., Ltd, Wuhan 430010, China - (2044414,727333041)@qq.com \\ ${ }^{2}$ School of Ordnance Non-commissioned Officer, Army Engineering University of PLA,Wuhan 430075,China- 77680756@qq.com \\ ${ }^{3}$ College of Remote Sensing and Geometics Engineering, Nanjing University of Information Science \& Technology, Nanjing, \\ 210044, China - pansy_nuist@163.com
}

Commission III, WG III/ IVc

KEY WORDS: Remote sensing, dynamic monitoring, water administration law, watershed management

\begin{abstract}
:
To realize real-time, detailed, and standardized watershed monitoring and management, a dynamic monitoring system is proposed, at all levels (space, air, and ground), by comprehensively utilizing advanced satellite and low-altitude unmanned aerial vehicle (UAV) technologies The system can be used to monitor and manage all kinds of sensitive water targets. This study takes water administration enforcement as an example for proving it feasibility by selecting typical study areas. This study shows that the proposed system is a promising information acquisition means, contributing to the development of watershed management.
\end{abstract}

\section{INTRODUCTION}

Watershed management means all kinds of country-conducted management activities aimed at the sustainable distribution of its resources and the process of creating and implementing plans, programs, and projects to sustain and enhance watershed functions that affect the plant, animal, and human communities within the watershed boundary. The management activities include the development, utilization, treatment, allocation, saving, and conservation of water resources, as well as the prevention and control of floods and droughts. Strengthening watershed water management plays a significant role in the long-term development of national economy and becomes a world-wise trend of river management (Cai 2009). Watershed management requires a long-term and dynamic monitoring within watershed ranges where key reservoir dynamic management is one of the essential tasks. Reservoir management involves floods control safety, water resources protection, water resources management, river management, water conservancy projects, soil and water conservation, and many other aspects. However, the reservoir management task is tedious, labour-intensive, policy-oriented, and strongly relying on professional knowledge and skills (Tan 2004; Yu 2010). It is difficult to provide effective supervision only by traditional manual inspections and reports from masses. Remotely sensed images can be widely used in reservoir management tasks (e.g., shoreline changes, illegal occupation, and occupation of water conservancy facilities) due to its advantages, such as, direct imaging and large coverage (Liang et al., 2010; Tang et al., 2009). Therefore, by combining advanced satellite, and lowaltitude UAV remote sensing technologies, and on-site inspections, an integrated dynamic monitoring system (at the space-air-ground levels) is carried out to obtain long-term change information by monitoring important water targets. The thematic change information (e.g., location distribution, spatial form, attributes, and other aspects) is useful for relevant

* Corresponding author management information systems. The method, through thematic analyses, can timely find hidden problems and provide scientific basis for watershed management to make decisions.

\section{THE SYSTEM}

\subsection{Data Acquisition}

A space-air-ground data acquisition system is a high-accurate, efficient integrated system that includes satellite remote, lowaltitude UAV, and ground-based sensors. The advantages of this integrated data acquisition system are listed as follows.

1) space-based satellite remote sensors provide multi-resolution, multi-temporal, multi-band, and multi-level remote sensing images, which, using as the main information sources, contribute to timely and remotely dynamic monitoring and management for large-coverage reservoir areas.

2) Low-altitude remote sensing technologies are mainly applied to daily monitoring tasks, and deal with some situations (such as, data missing and emergency data collection) due to weather caused blind areas.

3) Ground-based sensors are performed as a routine, periodic inspection tools, which particularly check some suspected targets and acquire their needed, complete attribute information.

\subsection{Data Processing}

According to the requirements of surveying authorities for monitoring scopes and objectives, thematic information extraction is an indispensable step. The detailed requirements can be founded as follows.

1) With the assistance of professional remote sensing image processing and geographic information system software, the 
collected multi-platform remote sensing data are first analysed and processed to obtain dynamic thematic information of the monitored objects based on spectral and spatial interpretation.

2) Field surveys are conducted on key target areas. Field measurement techniques are used to obtain high-accurate spatial locations, scale information, and related attribute information of the objects to be monitored.

3) By combining with the established thematic information and field measurement data, the monitored targets will be further analysed to obtain their accurate target information for relevant management departments.

\subsection{Information System}

Relying on the network and practical necessities of the supervision tasks, a dynamic monitoring information system is established. This system, driven by highly-accurate spatial data, is based on spatial information, dynamic monitoring information, and management service attribute data. The system, include three layers: 1) data support layer for data construction and storage, database information management system development, 2) middle service layer, and 3) application system layer.

The three layers are detailed as follows.

\section{(1) Data Support Layer}

In the data support layer, the data come from the following sources: the monitoring objects' interpretation results, basic geographic information data in the surveyed regions, and the field verification data.

\section{(2) Middle Service Layer}

The middle service layer, consisting of data access service, GIS map service, and GIS function service, are supported by basic information resource management and GIS platforms, respectively. Among these services, the GIS map service can be used for spatial data (vector and grid) services, attribute data services, (two-dimensional) map services, and (threedimensional) spatial data analysis services.

\section{(3) Application Layer}

The application layer includes three subsystems, i.e., monitoring information extraction, information supervision maintenance, and information supervision service. Both the monitoring information extraction and information supervision maintenance subsystems are Client/Server architecture operated by professional technicians. The information supervision service subsystem is Browser/Server architecture, where administrators can submit requests through the browser, and collect the results through data processing at the middle service layer.

The overall technical framework is shown in the following figure.

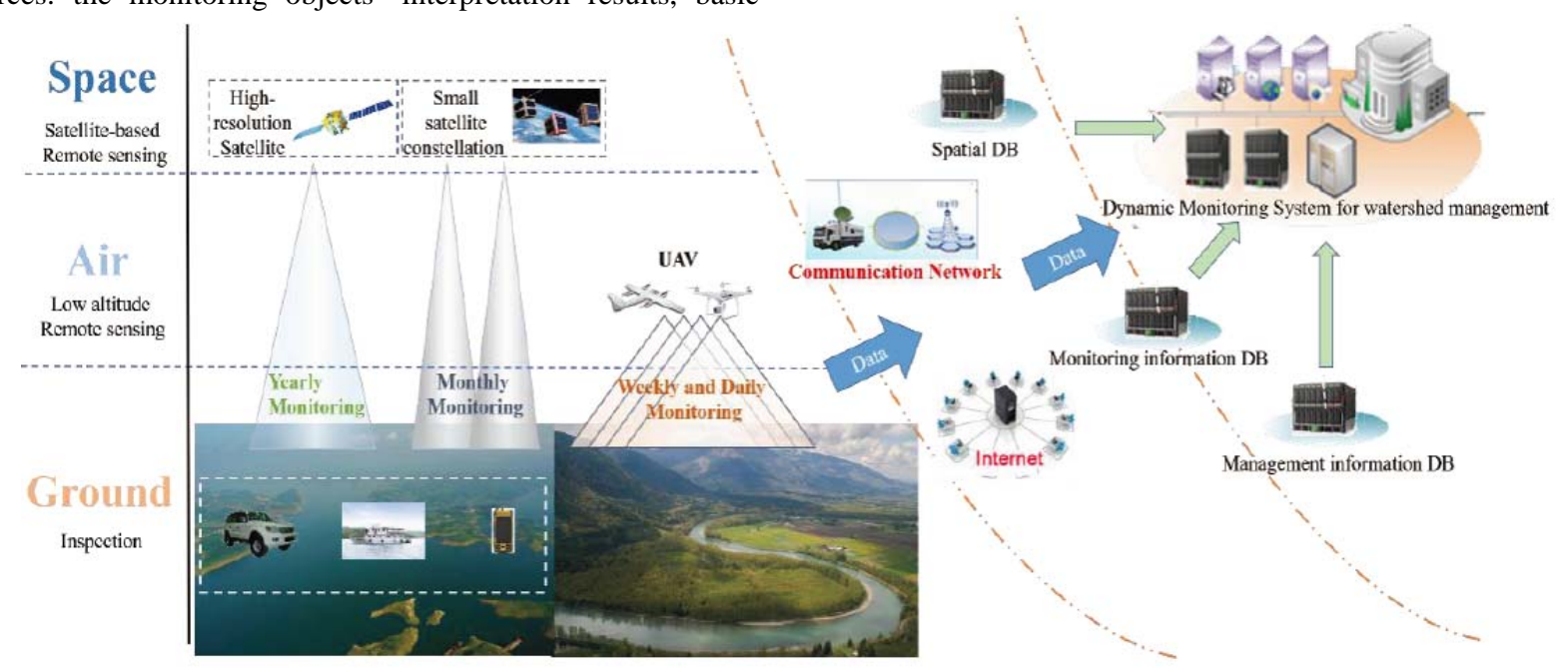

Figure 1. Dynamic monitoring system for watershed management based on space-air-ground co-observation

\section{KEY TECHNICAL ISSUES}

In the proposed monitoring information system, some key issues are detailed as follows.

\subsection{Monitoring Objects Determination and Data Acquisition Method Selection}

Different monitoring targets requires different spatial resolution and temporal resolution of remotely sensed data to be used. For example, water-related targets include hydropower stations, shipyards, factories, wharfs, water intakes, sewage outlets, spoil, waste soil and residue, construction sites, and other water types with different size and types. In addition, the monitoring timeliness also exist large differences. Therefore, it is necessary to select the appropriate data acquisition method for different types of monitoring objects.

1) High-resolution satellite remote sensing based monitoring. This monitoring system mainly monitors objects with long-term changes at smaller spatial scale. The remote sensing satellites with a spatial resolution smaller than 1 meter are selected as data sources. However, High-resolution satellite remote sensing based monitoring is difficult to rapidly cover monitoring areas due to their long re-visit period. Therefore, the system is appropriate for monitor objects of interest with the period of half-year.

2) Small satellite constellation based rapid monitoring. This monitoring system mainly monitors objects with short-term 
changes at larger spatial scale. To consider time-cycle requirements of monitoring targets, small satellite constellations can be used as data sources at the expense of certain spatial resolution. The spatial resolution of such satellites is taken into account (3 - 4 meters), the satellite images are difficult to be interpreted for accurately monitoring objects. Therefore the system is appropriate to for monitoring areas at a monthly cycle to quickly collect the changes of monitoring objects at the larger spatial scale.

3) Key-area on-demand monitoring. Because satellite remotely sensed data are limited by resolution, data observation windows, re-visit periods, and so on, real-time observation and analysis of certain specific monitoring objects are impossible to realize. Therefore, on-site inspection methods are essential for monitoring high timeliness requirements. For some monitoring targets with small sizes and occlusion, low-altitude remote sensing technologies, such as, mobile patrol equipment, patrol vehicles, patrol ships, are mainly used to carry out long-term, detailed monitoring tasks. To realize real-time tracking of the targets to be monitored, weekly, daily, even hourly monitoring might be required under special requirements or emergency conditions.

\subsection{Detailed Information Acquisition Mechanism}

Remotely sensed images are an important tool for collecting watershed information. In practical supervisory tasks, accurate plane and elevation data are essential. These accurate data requirements rely on comprehensive remote sensing interpretation and highly-accurate field measurement methods.

Because the geometric position information of the objects to be monitored obtained from remotely sensed images is twodimensional, the objects' edge feature points extracted from images are used to calculate their geometrical information such as areas and lengths. However, in some scenes with complex terrain types, the planmetric information obtained from remotely sensed images might generates large positioning errors, particularly without highly-accurate control points. Therefore, the remotely sensed images are used to roughly locate the positions of the targets to be monitored. In practical survey tasks, the image interpretation results of the targets are used to guide accurate field measurement means for obtaining highlyaccurate, detailed monitoring information of the targets.

\section{TYPICAL MONITORING CASES}

\subsection{Three Gorges Reservoir Area}

To evaluate the feasibility of the proposed monitoring system, watershed areas in the Three Gorges Reservoir are selected for water administration. The monitoring target is regional flood storage capacity, namely the filling behaviour in the reservoir area below the water level of 175 meters.

Using historical topographic and geomorphological data within the reservoir area, the working area was within 175 water level line of the Three Gorges Reservoir Area. In October 2015, under the support of the space-air-ground integrated monitoring results, we carried out supervision tasks in Yichang - Wanzhou section, the key area of the Three Gorges Reservoir Area. 19 suspected wading construction projects were found. In April 2016, we carried out supervision tasks in Yunyang - Chongqing section, another key area of the Three Gorges Reservoir Area. 12 suspected wading construction projects were found. The investigation results were further confirmed and transferred to the relevant law enforcement of water administration departments. Compared to the previous supervision methods based on manual inspection, almost one month was saved. Figure 2 (a) shows the thematic map overlapping the satellite image, and Figure 2(b) shows the manual inspection result.

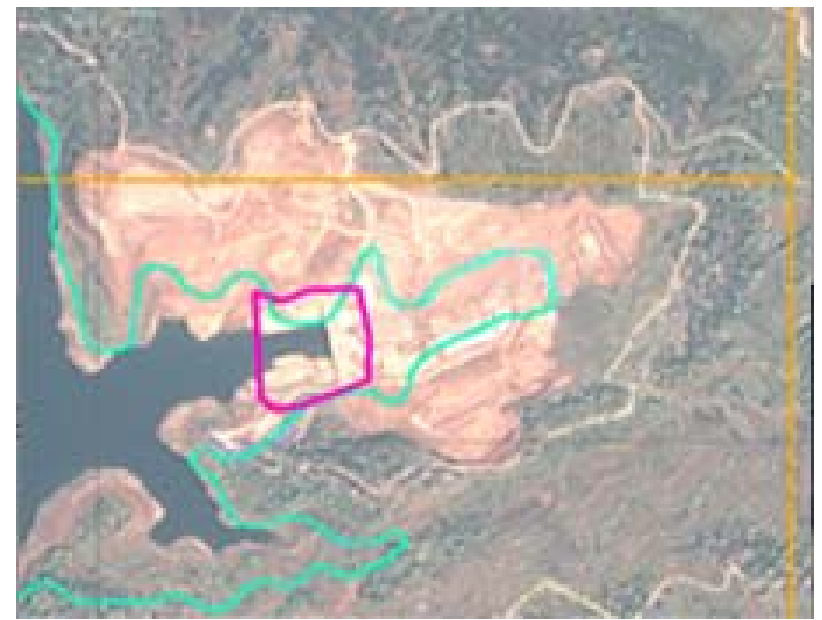

Figure 2 .(a)

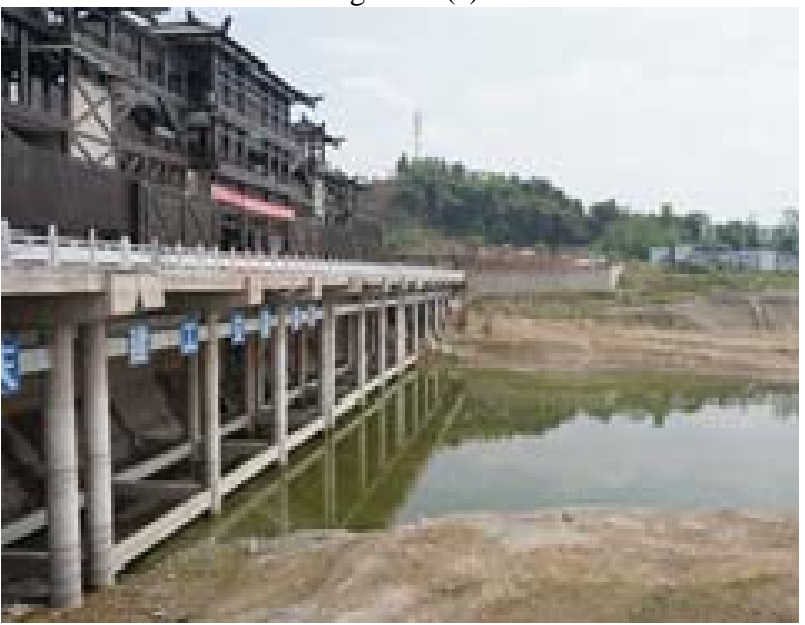

Figure 2 .(b)

Figure 2. an example in the Three Gorges Reservoir Area: (a) a thematic map overlapping the satellite image collected in 2016, and (b) the manual inspection result in 2016.

\subsection{Danjiangkou Reservoir Area}

Some small areas of the Danjiangkou Reservoir area were selected to demonstrate the performance of the proposed system. Similarly, the monitoring target is regional flood storage capacity, namely the filling behaviour, illegal setting wharf, sewage outfall, and bifurcatio dam construction embezzling storage capacity in the reservoir area below the water level of 170 meters.

With the support of the space-air-ground integrated monitoring system, 393 of the various construction projects were found in the Danjiangkou Reservoir Area in July 2014. More 22 suspected wading construction projects were found in June 2015 by the Danjiangkou detachment and the relevant local water administration departments. Compared to the previous supervision methods based on manual inspection, almost two months was saved. Figure 3 shows the operating window of the proposed system. Figure 4(a) shows the interpretation results 
using the satellite images, and Figure 4(b) shows the manual inspection results.

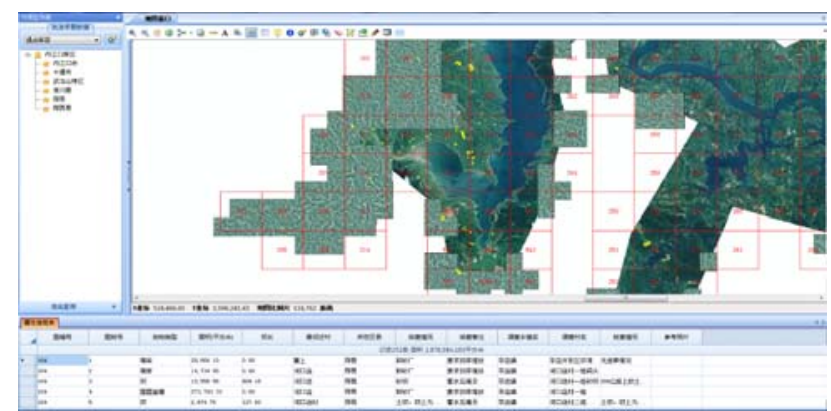

Figure 3. Monitoring information maintenance system

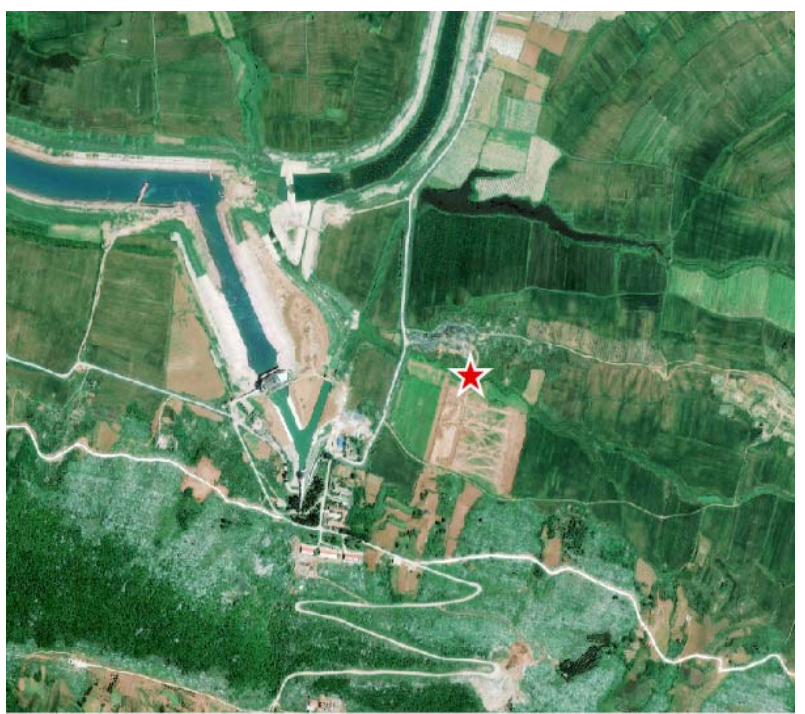

Figure 2 .(a)

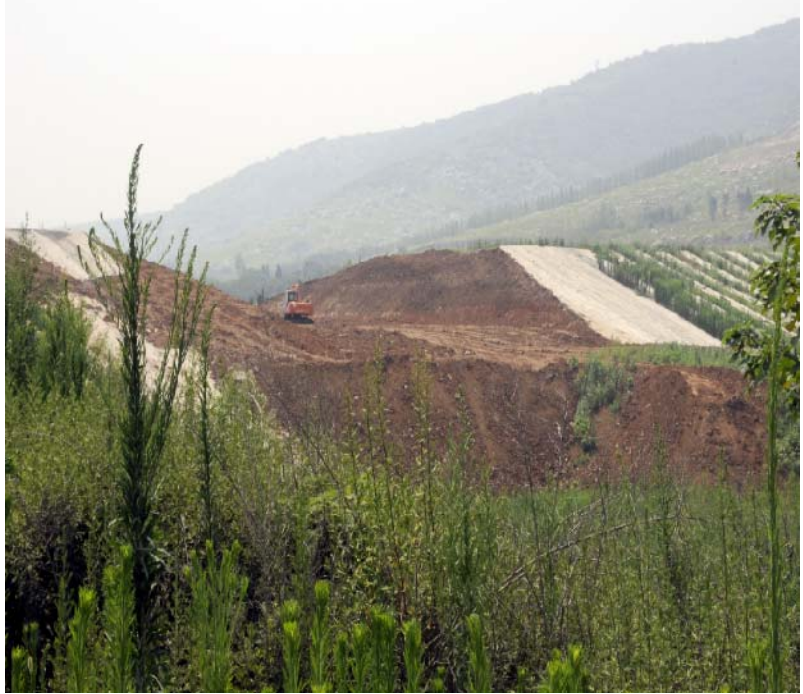

Figure 2 .(b)

(a) Interpretation results of high-resolution satellite images about non-conforming building dams, and

(b) manual inspection results.

\section{CONCLUSIONS}

This paper focuses on the analysis and discussion of the application in watershed monitoring based on space-air-ground co-observation and the description of data collection, processing, development of information system, and key technical issues in detail. The proposed dynamic monitoring system, integrating internal and external operations and highlyintegrated software and hardware, greatly reduces the cost of obtaining the monitoring target information for the watershed management agencies, leading to the improvement of the efficiency of targets to be monitored. The system has been proven to be feasibile through the practical application of law enforcement of water administration in typical monitoring areas of Yangtze watershed. The timely watershed change information provides data support for the maintenance of a good water order in the watershed, especially the key reservoirs, and is of great significance in advancing water management according to law, accelerating the strict management of water resources, and other aspects.

\section{REFERENCES}

Cai, Q., 2009. Strengthen River Basin Management for a Healthy Yangtze.Yangtze River, 08(1), pp.1-4.

Liang, D., Tan, D., Song, L., 2010. Application of Spatial Information Technology in Supervision and Law Enforcement of Water Administration. Journal of Yangtze River Scientific Research Institute, 27(1), pp.33-36.

Tan, X., 2004. A Study on Water administrative enforcement[D]. Tianjin University.

Tang, Q., Yu, S., Lu, J., 2009. Frame design of supervision of water administration information system in Zhujiang. PEARL RIVER, 30(2), pp.5-8.

Yu, S., 2010. Analysis on the existing problems and countermeasures in the current water administration law enforcement. Water Resources Development Research, 10(1), pp.54-55. 